\title{
Optical Tweezers for Quantitative Force Measurements and Live Cell Experiments
}

\author{
Philipp Rauch* and Torsten Jähnke
}

JPK Instruments GmbH, Bouchéstrasse 12, Haus 2, Aufgang C, 12435, Berlin, Germany

*rauch@jpk.com

\section{Introduction}

Optical tweezers employ light to manipulate objects. This manipulation takes place on the microscopic scale, allowing interrogation of small objects such as individual cells, cell compartments, single nanoparticles and (bio)molecules. Optical tweezers have a successful history starting from the first attempts to stably trap microparticles with dual counterpropagating beam setups in the early 1970s by Arthur Ashkin and colleagues [1]. Instrumental developments since that time [2-6] have led to the sophisticated turnkey systems of today. Up to now, optical tweezers have been mostly used by physicists able to design and construct an instrument from scratch. JPK has developed the NanoTracker ${ }^{\mathrm{TM}}$ 2, a compact, table-top instrument [7] that brings the technology to a broader range of scientists.

What are optical tweezers? The forces generated in an optical trap stem from the change of direction that tightly focused laser light undergoes when it is refracted at the interface of two optically different materials. The basic geometry is shown in Figure 1 where a spherical particle $\left(\mathrm{n}_{\text {particle }}>\mathrm{n}_{\text {medium }}\right)$ is placed in a focused beam that establishes a gradient of light intensity with cylindrical symmetry. Photons hit the particle surface at an angle and are deflected toward the vertical optic axis. Their momentum changes are transferred to the particle in the opposite direction. When the particle is displaced from the trap center, it is no longer in a stable position within the symmetric intensity gradient potential, and more momentum is transferred on the side facing the trap center. Thus, so-called gradient forces arise that drive the particle back to the trap center. Backscattering of photons gives rise to a second force pointing in the direction of light propagation that superimposes on the gradient force. As long as the intensity gradient is strong enough, for example, by the use of high numerical apertures, the gradient forces exceed the scattering force and the particle is stably trapped.

Developing a user-friendly system. We have developed an optical tweezers platform based on research-grade inverted optical microscopes and designed for sensitive manipulation, force, and tracking experiments. It provides the flexibility required by experienced users through open software and electronics architecture. As an off-the-shelf platform, the time-to-result for scientists can be greatly reduced; scientists can spend their time on experimentation rather than instrumentation. In this review, we will describe the criteria for building a user-friendly system that makes highly sensitive force measurements available to all scientific disciplines.

With over twelve years of effort developing and manufacturing scanning probe microscopes, JPK has accumulated experience in the field of high-precision scientific equipment. Knowledge of building nanometerprecise AFMs has enabled JPK to develop a commercial optical tweezers system with low drift and low mechanical noise that is capable of sub-piconewton $(\mathrm{pN})$ force measurements. The top-head design allows the smooth integration of conventional and advanced microscopy techniques for simultaneous use with optical tweezers, providing deeper insights into the systems under study. An important source for improvements to the original instrument has come from the experience of users employing it in a wide range of applications. Close cooperation with early-system users has led to an excellent knowledge exchange about the ideal operational requirements.

Optical trapping is an outstanding technique for direct single-molecule manipulations, force-measurements, and 3D tracking. For instance, to be able to track in great detail a single motor-protein moving a few $\mathrm{nm}$ steps or to quantitatively measure the establishment and rupture of single virus-receptor bonds, special attention must be paid to the design of the system to assure the quality of the trapping beam and its stability. In addition, the mechanical stability of the individual optical components, including lenses and filters, to trapping and detection objectives are important for sample drift stabilization.

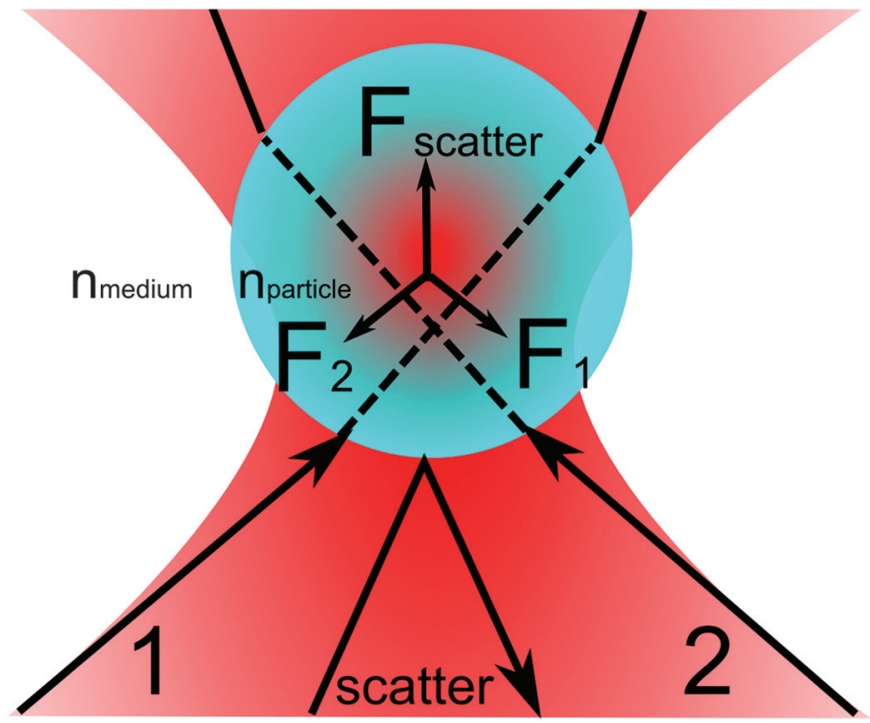

Figure 1: Principle of optical trapping. Light entering a particle with a different refractive index ( $\left.n_{\text {particle }}\right)$ than the surrounding medium $\left(n_{\text {medium }}\right)$ will change its direction and thus momentum. If the light is tightly focused and $n_{\text {particle }}>n_{\text {medium }}$, the transfer of momentum on the particle will drive it along the light intensity gradient toward the focus center due to the so-called gradient forces $F_{1}$ and $F_{2}$. In the trap center, these cancel out. Light scattering causes an additional force

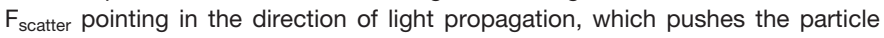
away from the focus. 


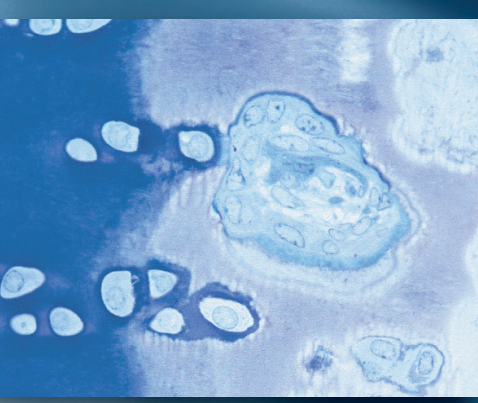

\section{DiATOME}

diamond knives

the highest quality...

the most precise sectioning... incomparable durability
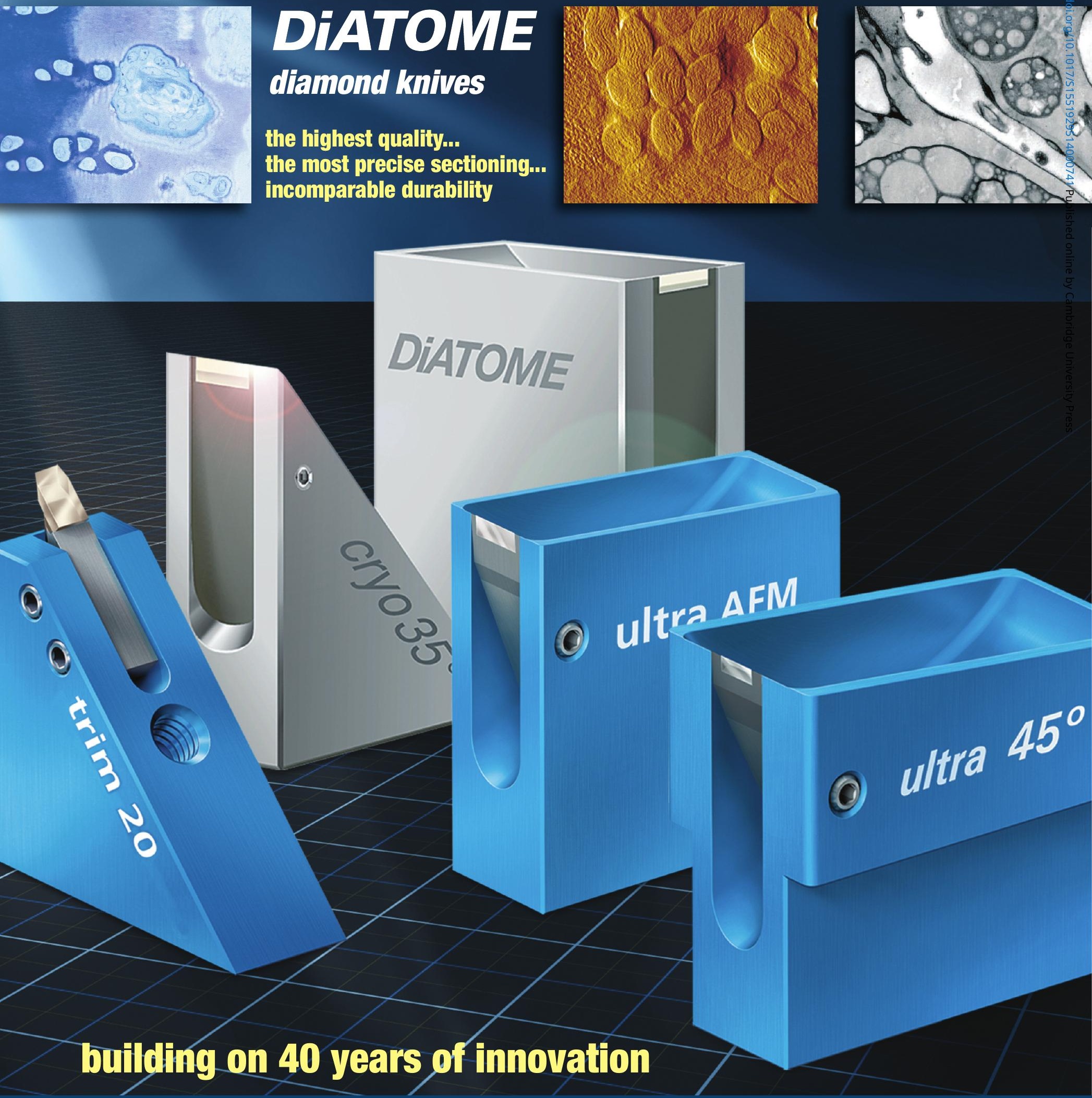

ultra $45^{\circ} \bullet$ cryo $~$ histo $\bullet$ ultra $35^{\circ}$ histo jumbo • STATIC LWN II • cryo immuno ultra sonic • ultra AFM \& Gryo AFM

NEWH.., trimtool 20 and trimtool 45

Finally, one trimming tool for all of your trimming needs, be it at room or cryo temperatures.
P.O. Box 550 • 1560 Industry Rd. • Hatfield, Pa 19440 Tel: (215) 412-8390 • Fax: (215) 412-8450 email: sgkcck@aol.com•stacie@ems-secure.com www.emsdiasum.com 
The key to versatile applications is modular and flexible design. Our design enables the system to be mounted on inverted research microscopes from Zeiss, Nikon, Olympus, and Leica. The addition of the head on top of the microscope does not block any of its optical ports, making it possible to use the whole range of standard high-end equipment including digital cameras, optical shutters, fluorescence filters, and illumination options. The modular design allows researchers to continue using well-established fluorescence techniques like total internal reflection fluorescence (TIRF) or confocal microscopy and, at the same time, benefit from having direct force sensing, tracking, and manipulation capabilities.

For ease of use and safety, the system is designed as a Class 1 laser product. This means that operation of the instrument does not require safety goggles, and no special training is needed for users. Because the device does not require a specially equipped laser laboratory, it suitable for multidisciplinary environments where optical tweezers may be used as a shared facility between biologists, chemists, and physicists.

\section{Designing a Practical System}

Particle manipulations. The system is designed to detect the smallest forces and manipulate particles or molecules with the highest precision. This means that detection of electronic noise and a stable laser source are crucial. Passive laser stabilization and specially designed detection electronics in the head provide low noise levels. Additionally, the compact folded design of the laser beam path makes the system immune to drift (Figure 2).

Double-beam or multi-beam configurations, combined solutions for coarse and precise sample positioning, and beam steering provide operational flexibility. In addition to extensive sample positioning control, the system combines the stability provided by a fixed trap with the flexibility of a moveable one. Moreover, the laser power can be controlled in a continuous manner for both traps independently. This freedom is required to allow a wide range of experimental assays and geometries. The two traps are available full time and are generated from a single laser source by polarization splitting, which makes the system ultra-stable against drift.

The new detection unit of the system is equipped with individual detectors for each trap, having separate diodes to capture lateral $(X Y)$ and axial $(Z)$ displacements of the trapped bead. Such a detection approach, in combination with softwarecontrolled dimming filters in front of each diode, allows the use of the full dynamic range of the detectors, achieving high sensitivity for various selected bead types, laser intensities, and trap split ratios.

Important for exact force measurements are precise calibration of the traps, lowest positional noise, and a flat trap stiffness profile over a large field of view. The one-button trap calibration procedure combines active oscillation with passive thermal noise analysis and is independent from bead size and medium viscosity. Furthermore, the cross talk between trap signals in detection is significantly reduced allowing interference-free signal acquisition even when both traps overlap.

Fast, low-noise electronics. The electronics unit controlling the optical tweezers system is optimized to perform at low noise levels with the highest possible bandwidth. Data acquisition can be performed at up to $60 \mathrm{MHz}$ (16-bit); whereas, at lower sampling frequencies 18-bit and 24-bit A/D channels are available. The signal access module (SAM) on the front of the controller provides an easy-to-use interface for feeding in auxiliary analog or digital signals. In addition, all internal signals can be monitored from the SAM. Similarly, external equipment such as cameras (for example, EM-CCDs), spectrometers, and detectors (for example, PMTs or APDs) can be connected with analog signals or triggered using transitor-transitor logic (TTL) pulses. All electronic signals (trap signals, 
sample, or trap positions and several auxiliary channels) can be recorded and streamed to disk with high bandwidth using any of the available real-time oscilloscope panels.

\section{Single Molecule Mechanics with Optical Tweezers}

Spatial, temporal, and force resolution. Single-molecule mechanics and manipulation experiments are very demanding. To acquire data with the highest precision, the instrument setup must be designed with lowest possible noise level and the highest stability. All components are optimized for sub-nm, femtonewton, and $\mu$ sec resolution.

The system software has several built-in modes to reproducibly perform various standardized experiments. This includes a comprehensive force spectroscopy package with a proprietary software package for either standard pulling experiments or more advanced ones involving force ramps, force clamps, etc. The built-in calibration manager is used for online calibration of the force and displacement detection system. Standard calibration is based on an entirely passive measurement of the thermal noise in the trap and thus does not affect the sample.

Microscope integration. The NanoTracker 2 was designed to seamlessly integrate with research-grade inverted optical microscopes from Zeiss, Nikon, Olympus, and Leica and can typically be incorporated into an existing system. The system can be used with high-NA objective lenses. The mounting of the objectives has been modified from the microscope standard in order to optimize trapping stability. The optical coupling of a $1064 \mathrm{~nm}$ tweezers laser into the light microscope base is accomplished through an additional port designed by JPK. The optical filters used for coupling-in the trapping laser were chosen to keep the entire visible spectrum (400-900 nm) open for other optical microscopy applications. Importantly, the standard microscope filter wheel is not touched by the NanoTracker 2 design, so that fluorescence imaging can be performed simultaneously and independently of optical trapping. This opens a wide range of applications that require a combination of several optical microscopy techniques. Camera commands from various manufacturers are fully embedded in the data acquisition software, including those from Jenoptik and Andor Technology to cover standard fluorescence experiments up to high-end single-molecule fluorescence imaging applications.

\section{Live Cell Applications}

Force measurements and manipulation of objects inside cells. By combination of hardware components, innovative design, integration possibilities, available accessories, advanced detection approaches, and flexible user-friendly software, the NanoTracker 2 can perform a number of advanced live-cell experiments. The user is able to obtain reliable quantitative data such as bacteria- or virus-to-cell force probing, detection of protein-ligand binding events in the cell membrane, and viscoelastic properties of a cell via membrane tether pulling or rheology.

Complementary to force measurement applications, NanoTracker 2 can be used to directly manipulate cells with optical traps, which then allow cell sorting, deformation, relocation, and stretching experiments. It is also possible to measure the force and the trajectory of moving or escaping cells, such as sperms or bacteria. The system can also be used to manipulate particles or organelles inside of living cells with highest precision. All of the manipulation or force experiments can be done simultaneously with fluorescence imaging or optical spectroscopy.

Minimized drift for long-term stability. For long-time experiments, which are often required for live cells, or optical tweezing in combination with techniques like TIRF or confocal microscopy, the low sample surface drift and trap stability become crucial characteristics. Here, the integrated, drift-minimized, and closed-chamber design plays a significant role.

Transmission illumination techniques such as bright field (BF) or differential interference contrast (DIC) are provided to visualize cells in media. Different from normal condenser light sources, the NanoTracker 2 is equipped with a state-of the-art integrated LED illumination unit in standard Köhler configuration, which allows BF or DIC in transmission. Optional fluorescence excitation sources include arc lamps, lasers, and LED-based systems that can be used for multicolor imaging. The standard side and back ports of the inverted microscope provide the freedom to combine several measurement techniques or use different kinds of analyzers or detectors such as VIS or Raman spectrometers, different types of cameras, or single-photon counters (PMTs, APDs) at the same time.

Heater for live-cell experiments. The uncompromising requirement of nearly all live-cell experiments is suitable environmental control. This is fully satisfied in the NanoTracker 2 with the availability of several accessories and the closed top-head design. For cell cultures, users may work with conventional glass-bottom Petri dishes from all major suppliers. Experimental temperatures are software-controlled by a specially designed Petri dish heater (Figure 3), which includes possibilities for buffer exchange

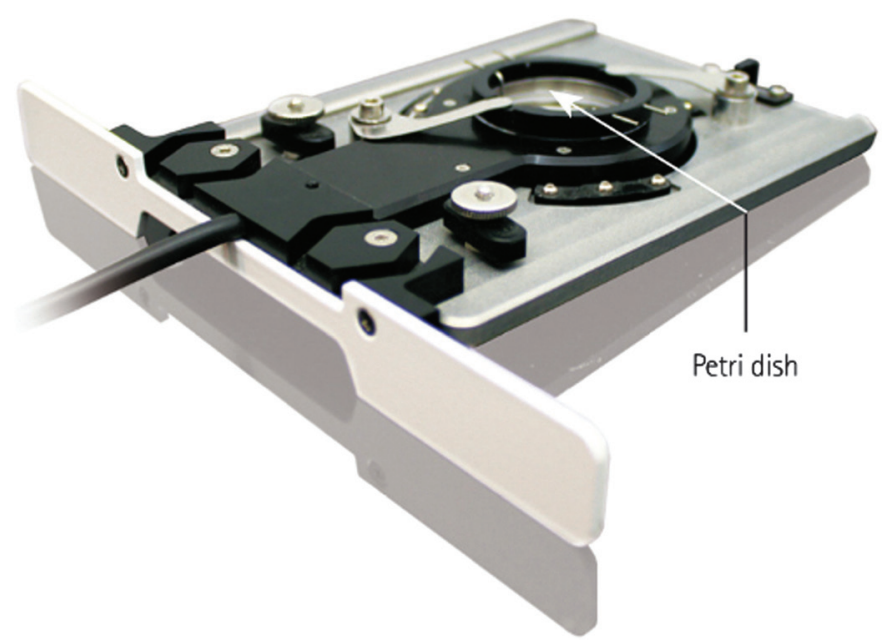

Figure 3: Heater for biological applications. Working with living cells, for example, in rheology or cell manipulation experiments, requires stable environmental conditions to ensure cell viability and a physiological environment. Today, many easy-to-use accessories for a broad range of experiments are available, like microfluidic systems or the displayed Petri dish heater for stable and precise temperature control. 

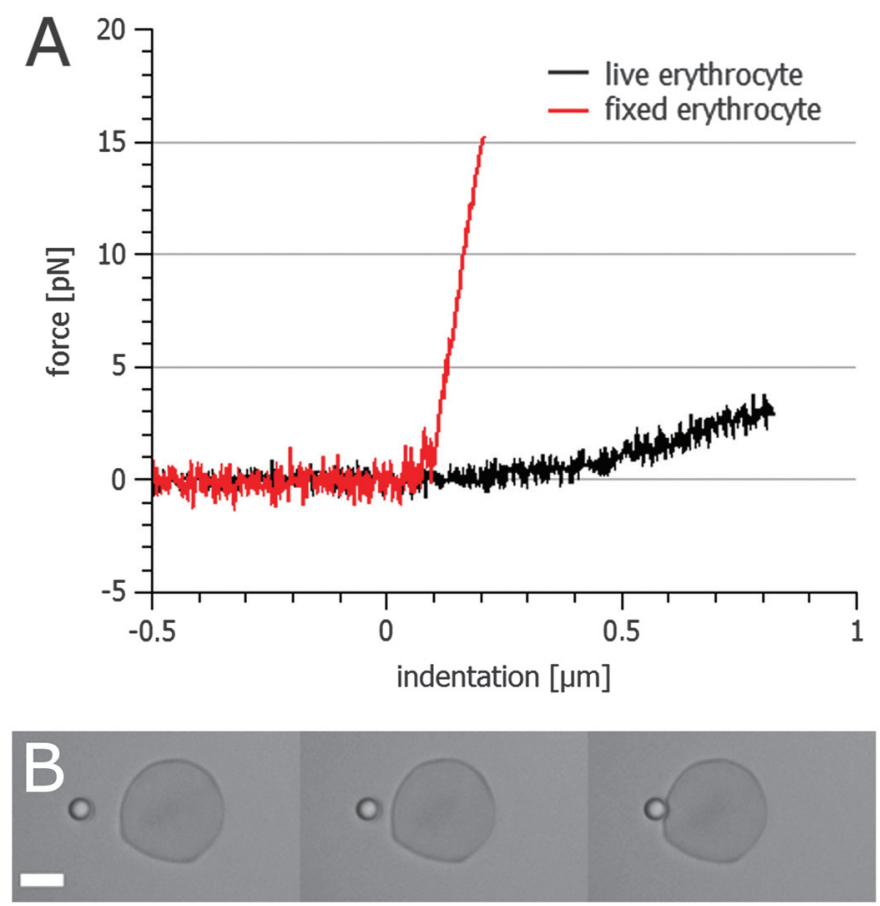

Figure 4: Erythrocyte elasticity measurements. Information about a cell's elasticity can be gathered in so-called indentation experiments. A polystyrene micro-bead is trapped and calibrated to allow high-resolution force measurements. Then a cell (here a red blood cell) is indented with the bead while the required forces are recorded. (A) The black curve shows the force-indentation relation for a living erythrocyte. An indentation depth of approx. $800 \mathrm{~nm}$ only requires forces as low as $3 \mathrm{pN}$. After chemical fixation with paraformaldehyde, the cell significantly stiffens as indicated by the much higher slope of the red curve (approx. $15 \mathrm{pN}$ for $150-200 \mathrm{~nm}$ of indentation). (B) Micrographs illustrating the course of the experiment show how the optically trapped particle is approached to a red blood cell (erythrocyte). Scale bar $=3 \mu \mathrm{m}$.

and a $\mathrm{CO}_{2}$ atmosphere. The PetriDishHeater ${ }^{\mathrm{TM}}(\mathrm{PDH})$ allows control of the temperature of the medium, as well as gas perfusion of samples mounted within Petri dishes. In the open geometry of such a Petri dish, a water-dipping objective lens is the detection objective of choice.

Rheology and micromechanics applications. System electronics and flexible hardware options enable rheological studies on living cells under optimal conditions. The rheological behavior of a wide range of materials is accessible because of the broadband electronics of the system. These enable the collection of bead movement spectra at up to $3.5 \mathrm{MHz}$. Whole-cell rheological experiments provide interesting insights into cellular mechanics. With the optical trap, nano-indentation measurements, as they are known in the atomic force microscopy community, can be performed with even higher precision and lower forces and are not limited to substrate-bound cells.

Suspended cells can be held fixed with multiple traps during force-indentation measurements using the multiplexing option. The spherical geometry of the indenter particles used here facilitates the analysis of the data and yields precise information on the mechanical response of biomaterials to forces in the piconewton range (Figure 4). From this type of measurement, conclusions can be made about the condition of cells in terms of blood diseases or various types and stages of cancer.

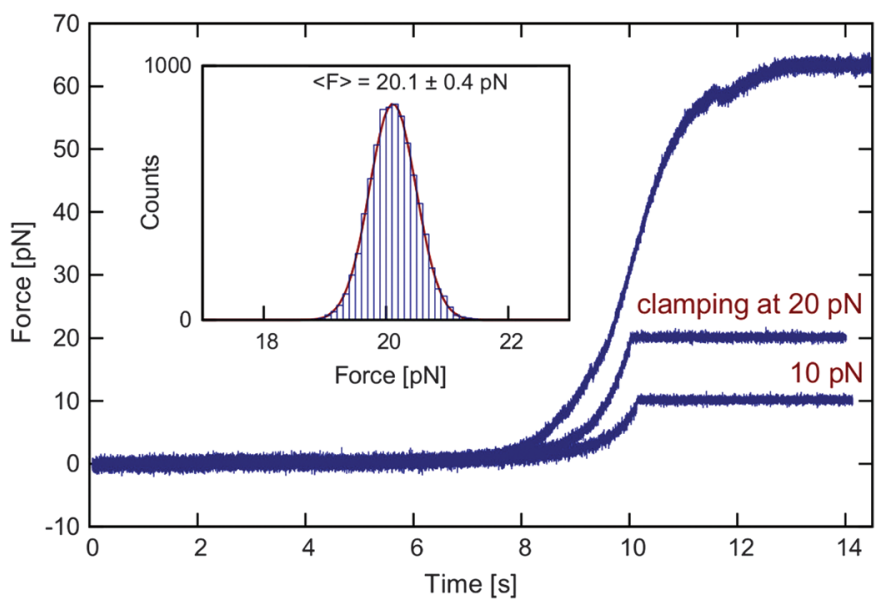

Figure 5: Force clamping and stretching of DNA molecules. Mechanical properties of DNA molecules play a role in many biological processes. Mounted between two trapped particles, it is possible to apply well-defined forces to the molecule and investigate its behavior. Modern optical tweezers setups feature so-called force-clamping where an active feedback loop is used to maintain a constant mechanical stress on single molecules. The three curves show clamping at $10 \mathrm{pN}$ and $20 \mathrm{pN}$ as well as (over-)stretching of a DNA molecule (from bottom to top). The small standard deviation of the force distribution shown in the histogram (inset) denotes the high stability and accuracy of the feedback system. Sample courtesy of Dr. Y. Jie's lab, University of Singapore.

Active tracking. Passive 3D tracking and active tracking (also called force- or position-clamping) are two of the main tools used to investigate processivity and force generation of motor proteins, cell membrane trafficking, binding events, and DNA-polymerase interactions. The force-clamping feature of the system allows feedback-assisted tracking to record the movements of single molecules or live cells with nanometer precision applying minimal, well-controlled forces.

Interactions between DNA and associated molecules are often accompanied by conformational and mechanical changes in the double-stranded helix causing tension or length changes of the molecule. These minute changes can be monitored if the molecule is stably held with two reporter beads attached to its ends using position- or forceclamp feedback systems. In the former case, the length of the molecule is kept constant at a slightly stretched configuration while the forces required to maintain this elongated state are recorded. In the latter case, a well-defined constant force is applied to the DNA strand, and changes in the contour length are followed through the feedback mechanism.

High-speed feedback loops implemented in the NanoTracker 2 system can be used to maintain defined tension levels with high accuracy. Figure 5 shows two force-time curves of DNA being stretched with constant forces of 10 and $20 \mathrm{pN}$, respectively The histogram shown in the inset illustrates the high accuracy and extremely small standard deviation of the applied force. The set value of $20 \mathrm{pN}$ is matched with a difference of only $0.1 \mathrm{pN}$ and a standard deviation as low as $0.4 \mathrm{pN}$. This allows the measurements of sub-pN forces generated by molecular binding events and interactions between DNA and related functional molecules. 


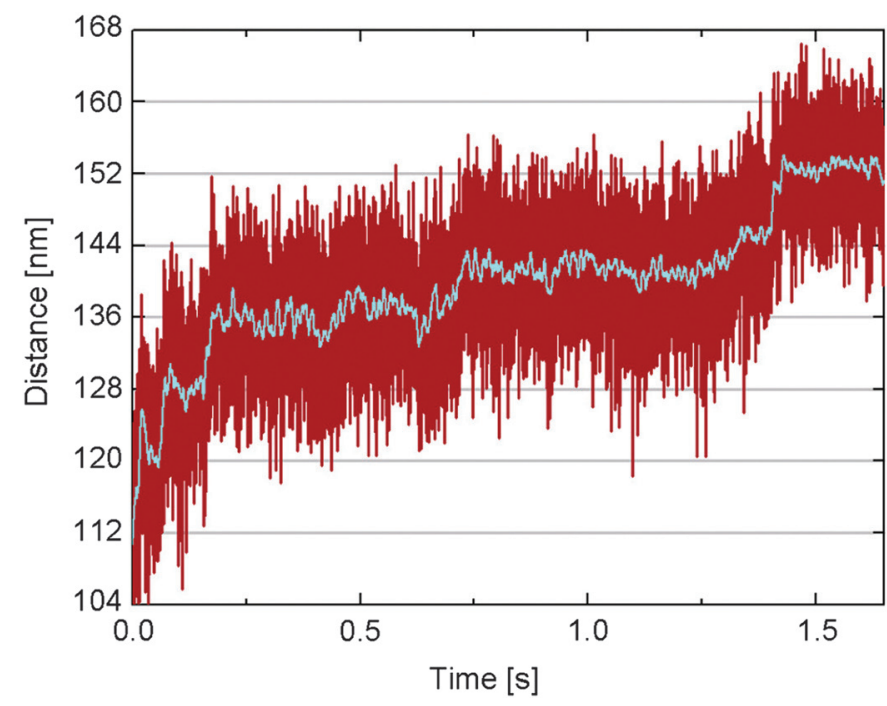

Figure 6: Watching motor proteins at work. Experiments fully utilizing the capabilities of state-of-the-art optical tweezers systems include the investigation of individual motor proteins as they are involved in intracellular transport processes. These proteins are able to move along cellular scaffolds in tiny steps carrying cargo around the cell. For the first time, optical tweezers allowed visualizing the stepping of such a motor. The curve shows the position of a kinesin motor protein tracked with optical tweezers over a longer period of time. The stepsize is approx. $8 \mathrm{~nm}$. Sample courtesy of Dr. V. Bormuth, MPI-CBG Dresden, Germany.

The versatile force clamp method can also be used to track the motion of reporter particles attached to motor proteins such as myosin, dynein, or kinesin that walk along intracellular filaments. For this tracking mode, only small laser powers and forces are required because the motion of the protein should not be obstructed. With highly sensitive quadrant photo diodes, these weak signals can be precisely detected, and the trajectory of the protein can be recorded. A closed loop feedback controls a piezo-driven stage and readjusts the sample position so that the bead and thus the protein remain in the center of the trap. Analyzing the feedback data delivers precise information about the motor protein motion. Figure 6 displays the position signal of a kinesin motor protein walking along a single microtubule. The characteristic step size of approximately $8 \mathrm{~nm}$ is well reflected in the data. With increasing laser power, the stall force, that is, the threshold load, which inhibits motor protein stepping, can also be determined.

Multiplexing. Some applications like the condensation of the bacterial chromosome or tracking dual-head motor proteins require an optical tweezers with four and more independently controllable optical traps. Trap multiplexing allows sensitive control of multi-molecular complexes. The ultra-fast switching of the laser beam from one trap position to the next is called beam sharing. Up to now this well-known technique had the drawback that it could not be combined with high sensitivity back-focal-plane interferometry detection. The high-speed data acquisition electronics of NanoTracker 2 allows signals from multiple traps to be time-resolved and interpreted separately. With this innovative de-multiplexing feature, the system is capable of high accuracy force measurements with up to eight independent traps generated from a single laser beam.

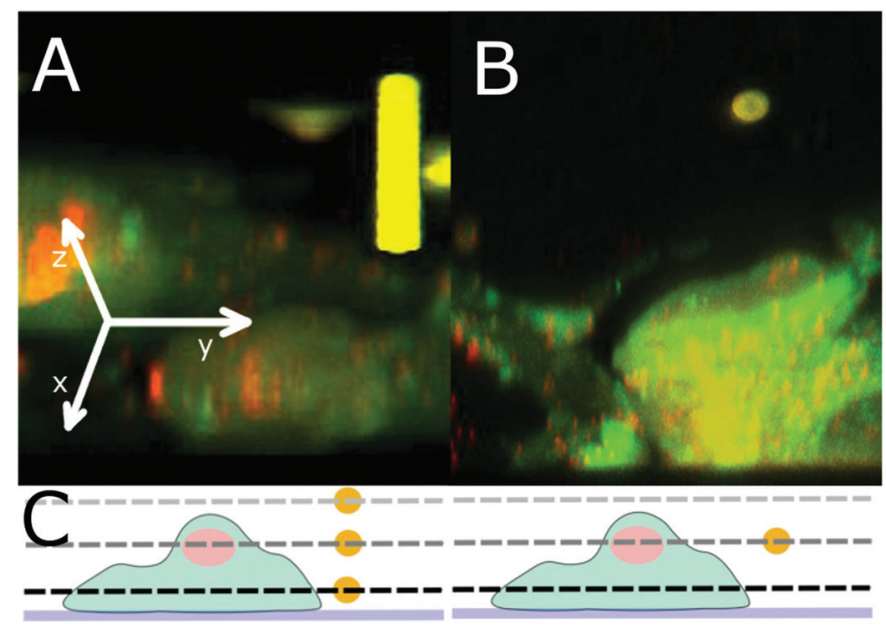

Figure 7: Simultaneous optical trapping and confocal imaging. Optically advanced systems allow integrating high-resolution fluorescence techniques like confocal laser scanning microscopy (CLSM) with optical trapping. Complex high-precision optics make the manipulation of trapped objects independent from 3D imaging. (A) 3D reconstruction of a confocal $z$-stack showing a GFP-labeled cell and a bead that moves with the imaging plane (represented by a column). (B) The same situation as in A with a bead that is optically manipulated to maintain a stable position relative to the cell during the recording of the $z$-stack.

Combination with confocal and fluorescence microscopies. The ability to combine optical tweezers with confocal microscopy becomes decisive in studies involving tissues, cells, and their internal compartments such as organelles. Confocal microscopy often is the only microscopy method that allows localization of regions of interest within a cell. Moreover, the proper alignment of optical tweezers is guided by fluorescence microscopy. None of the optical tweezers components block the confocal beam path.

Most internal cellular reactions to mechanical or biochemical stimuli are highly localized and require sensitive detection systems like confocal laser scanning microscopes (CLSM) to be resolved. Users can employ all the advantages of confocal microscopy with minimal sample and objective drift. Three-dimensional sample positioning with nanometer precision enables $z$-stacking and 3D sectioning of fluorescent samples. In addition, the optical traps can be shifted against the objective focus in the $z$ direction for particle manipulation beyond the imaging and scanning plane. As a result, a sample can be optically scanned in three dimensions with the trap either following the scan in $z$ or being held at a constant relative position to the sample. An example is shown in Figure 7 where in A the trapped bead moves with the scanning plane, whereas in B it remains stationary with respect to the sample cell. This opens access to a new generation of experiments where $3 \mathrm{D}$ manipulation and piconewton force measurements go together with high-resolution 3D fluorescence imaging. The investigation of cytoskeletal responses to mechanical manipulation is only one of many potential applications for this flexible type of setup.

\section{Conclusion}

The JPK NanoTracker 2 is an optical tweezers device that embodies the latest developments in beam-guiding technology including high-performance detection electronics, exceptional 
stability, and improved flexibility. This instrument is applicable for a broad range of experiments, particularly in cell biology, medicine, biophysics, and single-molecule mechanics. The main goal for this instrument was to make the techniques of optical trapping, force measurement, and particle manipulation accessible to scientists in a broad range of disciplines. The flexible NanoTracker 2 is complemented by several accessories for live cell environmental control, microfluidics, fluorescence microscopy, and multiple beam configurations.

\section{Acknowledgements}

We would like to thank Dr. Yan Jie (University of Singapore) and Dr. Volker Bormuth (MPI-CBG Dresden, Germany) for kindly providing samples of DNA and kinesin motor proteins that were used for the measurements shown in Figures 5 and 6.

\section{References}

[1] A Ashkin, Phys Rev Lett 24 (1970) 156-59.

[2] A. Pralle et al., Microsc Res Tech 44 (1999) 378-86.

[3] J Enger et al., Lab Chip 4 (2004) 196-200.

[4] JR Moffitt et al., Annu Rev Biochem 77 (2008) 205-28.

[5] O Otto, Rev Sci Instrum 79 (2008) 023710-.

[6] SNS Reihani et al., J Opt 13 (2011), 105301-1-105301-6.

[7] A Wozniak et al., Curr Pharm Biotechnol 10 (2009) 467-73.

[8] K Svoboda et al., Nature 365 (1993) 721-27.

[9] V Bormuth et al., Science 325 (2009) 870-73.

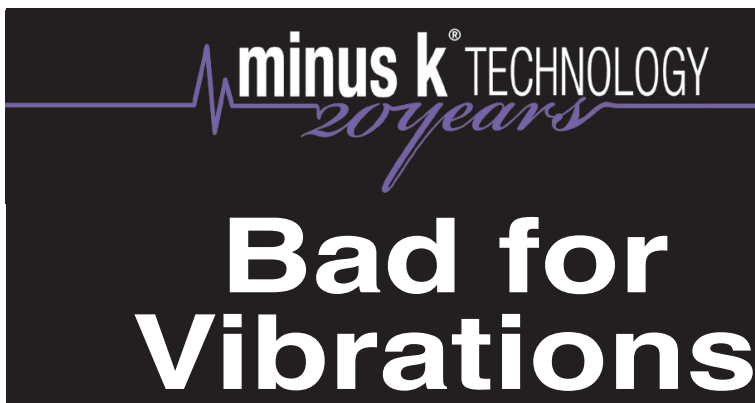

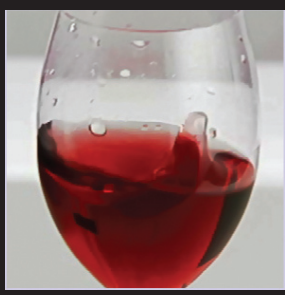

Without Minus $\mathbf{K}^{\circledR}$

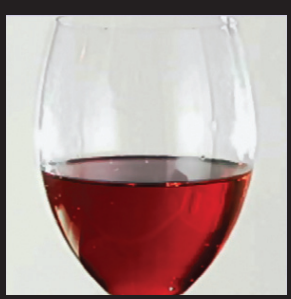

With Minus $\mathbf{K}^{\circledR}$

$$
\begin{aligned}
& \text { Great for } \\
& \text { Images }
\end{aligned}
$$

www.minusk.com

\section{THE NEW DESK V}

THE GOLD STANDARD IN SAMPLE PREPARATION

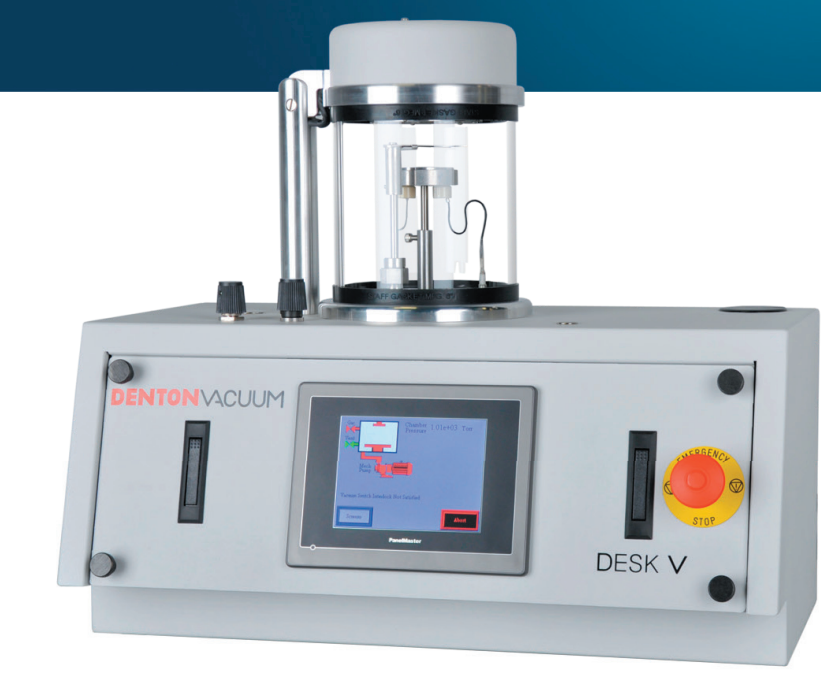

Now upgraded with storage for up to ten recipes and a color touch screen display, the Desk V HP delivers breakthrough electron microscopy sample preparation.

\section{DENTONVACUUM}

BARRIERS BECOME BREAKTHROUGHS

Visit us at:

www.dentonvacuum.com/mt

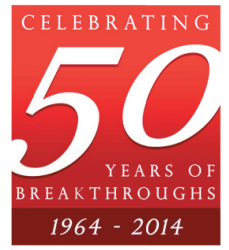


The moment "I think" becomes "I know".

\section{This is the moment we work for.}
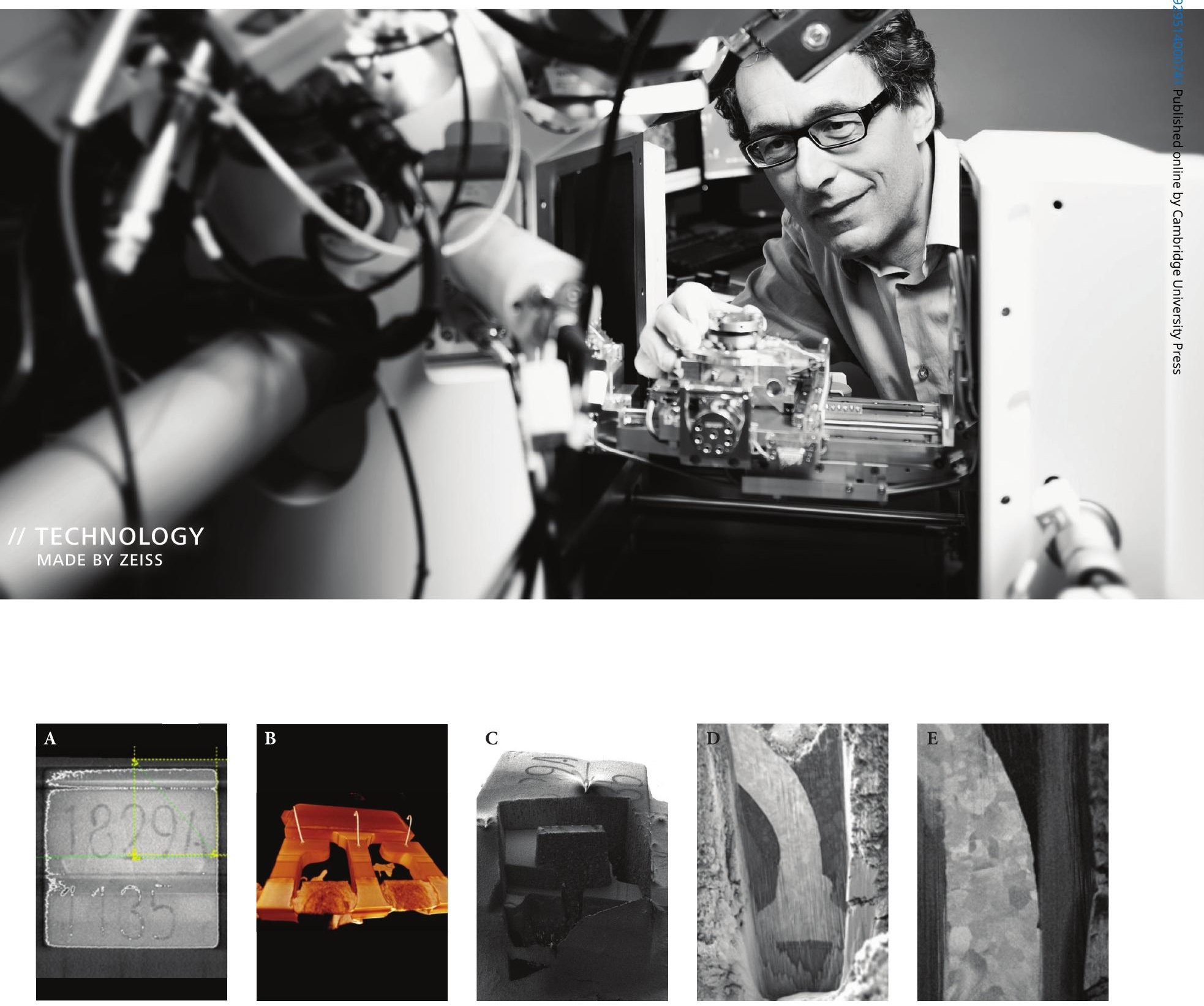

Correlative 3D-workflow: A) Hall sensor with internal defects, B) X-ray 3D image to localize bond wires and connections, C) Open sample with massive ablation laser and release Region of Interest, D) Fine polishing of defect structure using focused ion beam, E) HR imaging of defective area

With the unique portfolio of light, $X$-ray, electron and ion microscopes from ZEISS, you profit from optimized workflows for sample transfer and image correlation between the different systems. Use your ZEISS X-ray microscope to get structural information of your sample non-destructively in the micrometer scale, then, using your ZEISS FIB-SEM, precisely access the region of interest defined in your X-ray image to perform your high resolution imaging and analytics. For more information, visit us at M\&M in Harford, CT at Booth \#602, or contact us at info.microscopy.us@zeiss.com.

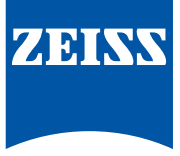

